\title{
INFLUÊNCIA DO DESGASTE DA ESFERA NA FORMAÇÃO DAS CRATERAS DE DESGASTE EM ENSAIOS BALL-CRATERING
}

Ronaldo Câmara Cozza'

\section{Resumo}

Surgido no setor industrial, o ensaio de desgaste micro-abrasivo por esfera rotativa difundiu-se rapidamente no meio acadêmico, sendo utilizado em diversas pesquisas envolvendo desgaste abrasivo de materiais. Entretanto, um assunto pouco discutido entre os pesquisadores é o desgaste da esfera, que, dependendo da configuração do equipamento, pode fazer com que as calotas geradas apresentem contornos que divergem ao formato circunferencial. Consequentemente, essa imperfeição ocasionará erros quando forem calculados, além do próprio diâmetro, a altura e o volume da cratera de desgaste. Este trabalho apresenta um estudo sobre a influência do desgaste da esfera na formação das crateras de desgaste em ensaios de desgaste micro-abrasivo por esfera rotativa fixa. Foram utilizados corpos-de-prova de aço-ferramenta (HSS) M2, metal duro (WC-Co) classe P20 e uma esfera de aço AISI 52 I00. A lama abrasiva foi preparada com carbeto de silício $(\mathrm{SiC})$ preto (com tamanho médio de partícula de $5 \mu \mathrm{m}$ ) e água destilada. Durante os ensaios, a pasta abrasiva foi inserida de maneira intermitente entre o corpo-de-prova e a esfera, através de um conta-gotas. As crateras de desgaste foram analisadas por meio de um microscópio óptico, com a finalidade de verificar seus contornos. Foi observado que, até um determinado grau de desgaste apresentado pela linha de trabalho da esfera de ensaio, os contornos das calotas esféricas tendiam a uma circunferência. Conforme o desgaste da esfera foi aumentando, os contornos apresentaram formatos denominados "elíptico", "oblongo" e " 8 ". Além disso, com o aumento do nível de desgaste da esfera de ensaio, relatou-se um aumento do desvio-padrão dos valores dos diâmetros das crateras de desgaste.

Palavras-chave: Ball-cratering wear test; Desgaste abrasivo; Desgaste da esfera.

\section{BALL WEAR INFLUENCE AT THE CAPS FORMATION IN THE MICRO-ABRASIVE WEAR TESTING BY BALL-CRATERING}

\begin{abstract}
The micro-abrasive wear testing by rotating ball appeared in the industrial sector and quickly spread to academic institutions, where it has been used in many studies on the micro-abrasive wear of materials. However, ball wear is an issue that has received only little attention. Depending on equipment configuration, ball wear can result in craters with boundaries different from circumferential. Consequently, this imperfection will cause errors during the calculation, besides the diameter itself, on the height and the volume of the crater. This work presents a study on the influence of ball wear on crater formation in the micro-abrasive wear testing with fix ball. Tests were conducted with HSS M2 and WC-Co P20 specimens and one ball of AISI 52100 steel. The slurry was prepared with black silicon carbide (SiC) (with mean particle size of $5 \mu \mathrm{m}$ ) and distilled water. During the tests, the slurry was supplied intermittently between the specimen and the ball, by a dropper. The craters were analyzed by optical microscopy, in order to verify their boundaries. It was observed that, until a given wear level of the ball, the boundaries of craters tended to a circumference. When the ball wear level increased, the boundaries presented tendency for contours called "elliptical", "oblong" and "8" ("eight"). Besides, with the increase of the ball wear level, it was reported an increase of the standard deviation of the diameters values of the wear craters.
\end{abstract}

Keywords: Ball-cratering wear test; Abrasive wear; Ball wear.

'Departamento de Engenharia Mecânica, Centro Universitário Fundação Educacional Inaciana "Padre Sabóia de Medeiros" - FEl, Centro Estadual de Educação Tecnológica "Paula Souza" - CEETEPS, FATEC-Mauá, São Bernardo do Campo, SP, Brasil. E-mail: rcamara@fei.edu.br; ronaldo.cozza@fatec.sp.gov.br 


\section{INTRODUÇÃO}

O ensaio de desgaste micro-abrasivo por esfera rotativa é aplicado em pesquisas que abordam o comportamento de materiais metálicos [I-3] e não-metálicos [4-7], podendo ser aplicadas forças normais menores que $0,1 \mathrm{~N}[8,9]$ até $5 \mathrm{~N}[3,10]$ e, em ensaios conduzidos com filmes finos de espessura $2,5 \mu \mathrm{m}$, é possível obter calotas esféricas sem atingir o substrato [I I]. A Figura la apresenta o princípio deste tipo de ensaio, em que uma esfera é forçada contra o corpo-de-prova na presença de um composto abrasivo; na Figura Ib tem-se uma cratera de desgaste típica gerada, com a indicação do diâmetro "d" [12].

Para este tipo de ensaio, há duas configurações mecânicas de equipamentos [13]: i) "esfera-livre" e ii) "esfera-fixa", cada um com suas vantagens e desvantagens. Entretanto, com a adoção de equipamentos que possuem configuração "esfera fixa", conforme os ensaios vão sendo realizados, a esfera sofre, consequentemente, desgaste, mas, somente em um dos hemisférios. A magnitude desse desgaste é proporcional às durezas do corpo-de-prova, das partículas abrasivas e ao número de ensaios. Devido a esse desgaste, são geradas crateras com contornos que fogem do padrão "circular", inserindo erros nos cálculos das dimensões (diâmetro - $d$, profundidade $-h$ e volume $-V$ ) das crateras de desgaste.

Com isso, o objetivo deste estudo é apresentar os possíveis contornos das crateras de desgaste que são gerados devido ao próprio desgaste da esfera de ensaio.

\section{EQUIPAMENTOS, MATERIAIS E PROCEDIMENTO EXPERIMENTAL}

\section{I Equipamento de Ensaio de Desgaste Micro-Abrasivo por Esfera Rotativa Fixa}

Para os ensaios de desgaste micro-abrasivo, utilizou-se um equipamento de ensaio de desgaste micro-abrasivo por esfera rotativa fixa. A Figura 2 mostra seu princípio de funcionamento.
A força normal $(N)$ é responsável pelo contato entre a esfera e o corpo-de-prova. Durante os ensaios, o composto abrasivo é inserido entre a esfera e o corpo-de-prova.

\subsection{Materiais}

Os corpos-de-prova foram de aço-ferramenta (HSS) M2 (temperado e revenido) e WC-Co P20.

Para a análise da microestrutura do aço-ferramenta M2, um dos corpos-de-prova foi embutido em baquelita. Após o embutimento, a superfície do corpo-de-prova foi lixada, obedecendo a seguinte sequência de lixas: grão 220, 320, 400 e 600 . Em seguida, a superfície foi polida com pastas diamantadas de diferentes granulometrias, na sequência de 6,3 e $0,25 \mu \mathrm{m}$. A última etapa foi o ataque químico da superfície com Nital $3 \%$.

Assim como no aço-ferramenta M2, para a análise da microestrutura do WC-Co P20, um corpo-de-prova foi embutido em baquelita e lixado. Em seguida, o mesmo foi submetido ao processo de polimento. $O$ ataque químico da superfície foi realizado com MURAKAMI, composto por $10 \mathrm{~g}$ de ferrocianeto de potássio $\left(\mathrm{K}_{3} \mathrm{Fe}(\mathrm{CN})_{6}\right), 10 \mathrm{~g}$ de hidróxido de potássio $(\mathrm{KOH})$ e $100 \mathrm{ml}$ de água destilada.

Foi utilizada uma esfera de aço AISI 52100 temperado e revenido, de diâmetro $D=25,4 \mathrm{~mm}(D=\mathrm{I}$ "). Em uma segunda esfera, utilizada somente para a análise da microestrutura do aço AISI 52100 temperado e revenido, a preparação de sua superfície foi realizada da mesma maneira e com os mesmos materiais utilizados para o aço-ferramenta M2.

As Figuras 3a, 3b e 3c mostram, respectivamente, as microestruturas do aço ferramenta M2, do WC-Co P20 e do aço AISI $52100[1,2]$.

A pasta abrasiva foi preparada com carbeto de silício (SiC) preto, comercial de grau técnico, da Alcoa [14] e água destilada. A Figura 4a mostra uma micrografia eletrônica de varredura do abrasivo utilizado. A distribuição granulométrica desse material é apresentada na Figura 4b.

Para o cálculo da dureza do $\mathrm{SiC}$, uma porção do material foi sinterizada. Dez indentações Vickers foram conduzidas sob uma carga de $50 \mathrm{~N}$ e tempo de $15 \mathrm{~s}$ [14].

(a)

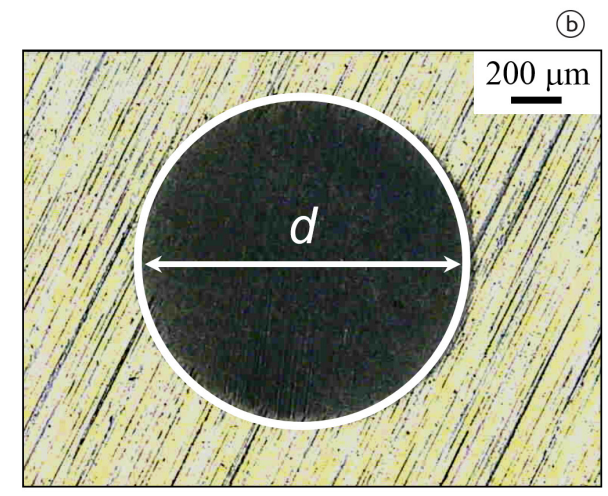

Figura I. (a) Princípio do ensaio de desgaste micro-abrasivo por esfera rotativa e (b) cratera de desgaste com a indicação do diâmetro "d" [I 2]. 
Com os mesmos valores de força e tempo $(50 \mathrm{~N}$ e I5 s, respectivamente) adotados por lzhevskyi et al. [14], foram medidas as durezas Vickers dos corpos-de-prova (HSS M2 e WC-Co P20) e das esferas de ensaio de aço AISI 52100. De forma aleatória, foram realizadas treze indentações na superfície de cada material. Após os ensaios, descartou-se o valor de dureza obtido na primeira indentação. Em seguida, a dureza do material foi determinada pela média aritmética dos dez valores mais próximos entre si.

A Tabela I reúne os valores das durezas dos materiais utilizados (corpos-de-prova, esfera de ensaio e material abrasivo).

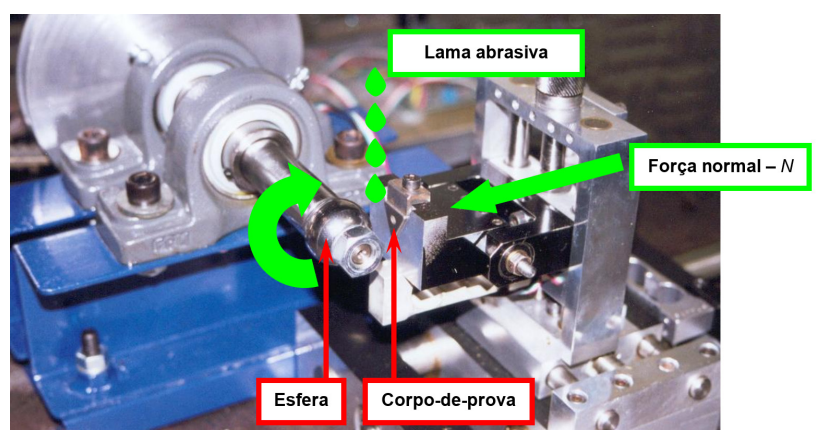

Figura 2. Equipamento de ensaio de desgaste micro-abrasivo por esfera rotativa fixa utilizado nos experimentos.

\subsection{Procedimento Experimental}

A Tabela 2 apresenta os valores dos parâmetros definidos para os ensaios.

A força normal definida para os ensaios de desgaste foi de $N=1,25 \mathrm{~N}$, junto a seis distâncias de deslizamento (S), $S_{1}=8 \mathrm{~m}, \mathrm{~S}_{2}=15 \mathrm{~m}, \mathrm{~S}_{3}=20 \mathrm{~m}, \mathrm{~S}_{4}=25 \mathrm{~m}, \mathrm{~S}_{5}=35 \mathrm{~m}$ e $S_{6}=40 \mathrm{~m}$.

Com base na distância de deslizamento, para os ensaios com os corpos-de-prova de aço-ferramenta $M 2$, foi estabelecido os seguintes grupos e a seguinte sequência de ensaios:

- $I^{\circ}$ Grupo de Ensaios $\Rightarrow S: 20,15,20,25,25,20,15$, I5, I5, 25, 25 e 20 m;

- $2^{\circ}$ Grupo de Ensaios $\Rightarrow$ S: 35, 40, 35, 8, 40, 8, 40, 8, $8,35,35$ e $40 \mathrm{~m}$.

Tabela I. Dureza dos materiais utilizados nos ensaios

\begin{tabular}{ccc}
\hline & Material & Dureza \\
\hline Corpos-de- & HSS M2 & $652 \mathrm{HV} 5$ \\
prova & WC-Co P20 & $1193 \mathrm{HV5}$ \\
$\begin{array}{c}\text { Esfera de ensaio } \\
\text { Partículas }\end{array}$ & Aço AISI 52100 & $856 \mathrm{HV5}$ \\
abrasivas & $\mathrm{SiC}$ & $1886 \mathrm{HV5}-1937 \mathrm{HV5}$ \\
\hline
\end{tabular}
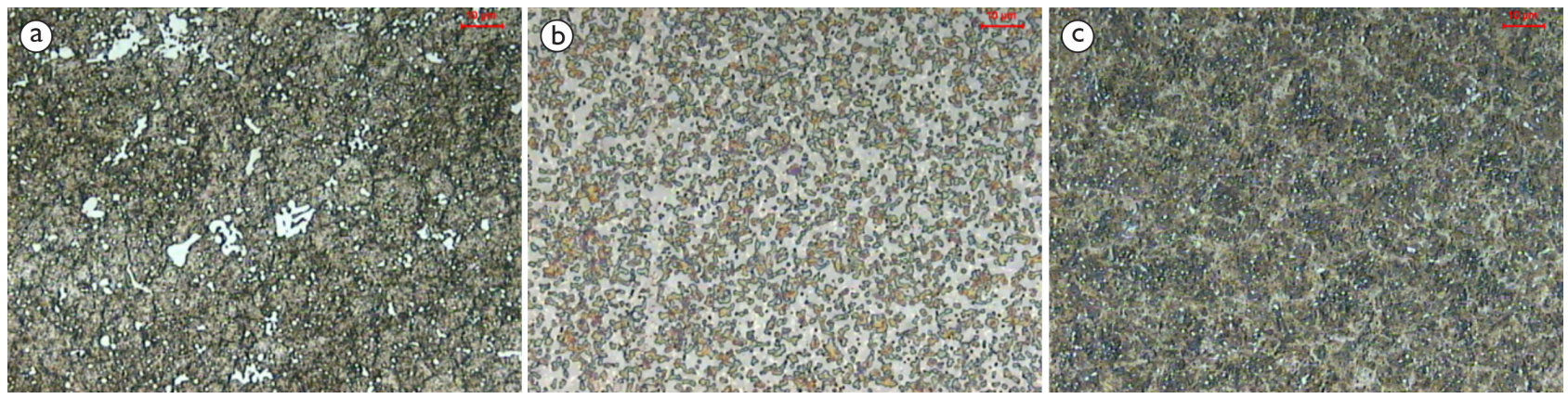

Figura 3. Microestruturas: (a) aço-ferramenta M2, (b) WC-Co P20 e (c) aço AISI 52100.
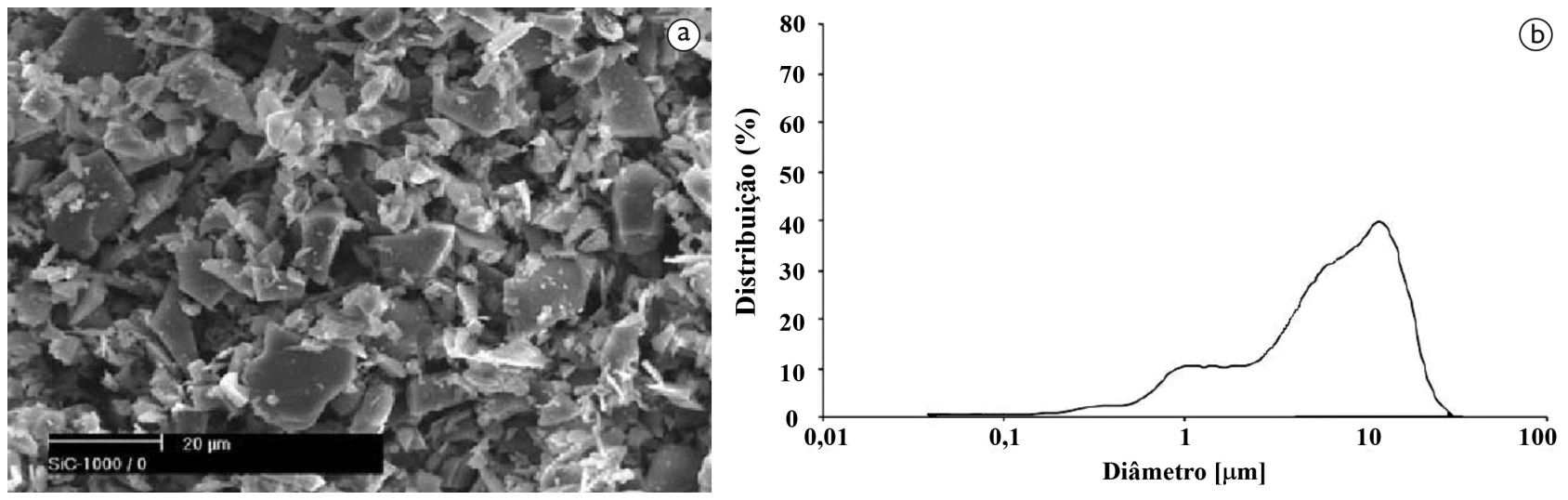

Figura 4. SiC [14]: (a) Micrografia eletrônica de varredura e (b) distribuição granulométrica. 
Tabela 2. Valores dos parâmetros estabelecidos para os ensaios realizados

\begin{tabular}{|c|c|c|c|c|c|c|}
\hline Condição de ensaio & $\mathbf{I}$ & 2 & 3 & 4 & 5 & 6 \\
\hline Força Normal - N [N] & 1,25 & 1,25 & 1,25 & 1,25 & 1,25 & 1,25 \\
\hline Distância de deslizamento $-S[\mathrm{~m}]$ & 8 & 15 & 20 & 25 & 35 & 40 \\
\hline Rotação da esfera - $n$ [rpm] & 37,6 & 37,6 & 37,6 & 37,6 & 37,6 & 37,6 \\
\hline Velocidade tangencial periférica $-v[\mathrm{~m} / \mathrm{s}]$ & 0,05 & 0,05 & 0,05 & 0,05 & 0,05 & 0,05 \\
\hline Tempo de ensaio $-t[\min \mathrm{s}]$ & $2 \min 40 s$ & $5 \mathrm{~min}$ & $6 \min 40 s$ & $8 \min 20 s$ & $1 \mathrm{I} \min 40 \mathrm{~s}$ & $13 \min 20 \mathrm{~s}$ \\
\hline Gotejamento da pasta abrasiva & I gota / $10 \mathrm{~s}$ & I gota / $10 \mathrm{~s}$ & I gota / $10 \mathrm{~s}$ & I gota / $10 \mathrm{~s}$ & I gota / $10 \mathrm{~s}$ & I gota / $10 \mathrm{~s}$ \\
\hline
\end{tabular}

Seguindo esse mesmo critério, para os ensaios com as pastilhas de WC-Co P20, os grupos e a ordem de ensaios estabelecida ficaram como:

- $3^{\circ}$ Grupo de Ensaios $\Rightarrow$ S: 8, 15, 8, 20, 15, 20, 15, 8, 8, 20, 20 e $15 \mathrm{~m}$;

- $4^{\circ}$ Grupo de Ensaios $\Rightarrow$ S: 40, 25, 25, 35, 40, 35, 25, $40,35,35,40$ e $25 \mathrm{~m}$;

- $\quad 5^{\circ}$ Grupo de Ensaios $\Rightarrow$ S: 15, 15, 35, 15, 35, 15, 35 e $35 \mathrm{~m}$.

A rotação da esfera foi de $n=37,6 \mathrm{rpm}$, mesmo valor adotado por Trezona et al. [3]. Com a esfera de diâmetro $D=25,4 \mathrm{~mm}$ tem-se uma velocidade tangencial periférica de $v=0,05 \mathrm{~m} / \mathrm{s}$, o que evita efeitos hidrodinâmicos [8].

Para as distâncias de deslizamento $S_{1}=8 \mathrm{~m}$, $S_{2}=15 \mathrm{~m}, \mathrm{~S}_{3}=20 \mathrm{~m}, \mathrm{~S}_{4}=25 \mathrm{~m}, \mathrm{~S}_{5}=35 \mathrm{meS}_{6}=40 \mathrm{~m}$, junto à $v=0,05 \mathrm{~m} / \mathrm{s}$ foram calculados os tempos de ensaio (t) $t_{1}=2 \mathrm{~min} 40 \mathrm{~s}, t_{2}=5 \mathrm{~min}, t_{3}=6 \mathrm{~min} 40 \mathrm{~s}, t_{4}=8 \mathrm{~min} 20 \mathrm{~s}$, $t_{5}=11 \mathrm{~min} 40 \mathrm{~s}$ e $t_{6}=13 \mathrm{~min} 20 \mathrm{~s}$, respectivamente.

Todos os ensaios foram realizados sem paradas intermediárias e uma gota de lama abrasiva foi inserida a cada $10 \mathrm{~s}$ entre a esfera e o corpo-de-prova.

\section{RESULTADOS E DISCUSSÃO}

\section{I Contornos das Crateras de Desgaste}

\section{I.I Contorno circular}

Nos ensaios realizados com os corpos-de-prova de aço-ferramenta $M 2$, praticamente todas as calotas esféricas geradas com o " $1 \circ$ Grupo de Ensaios" e o " $2^{\circ}$ Grupo de Ensaios" exibiram contornos tendendo a uma circunferência, como pode ser observado na Figura 5.

Quando a esfera está com um "baixo" nível de desgaste na linha de trabalho, o que irá formar a calota esférica no corpo-de-prova é o arco $\overparen{\mathrm{AB}}$, cujo raio $\left(R_{\mathrm{AB}}\right)$ é igual (ou, aproximadamente) ao raio da esfera $(R)$. Com isso, para $R_{A B}=R$ ou $R_{A B} \cong R$, o contorno da cratera de desgaste tenderá a uma circunferência, como é ilustrado na Figura 6.

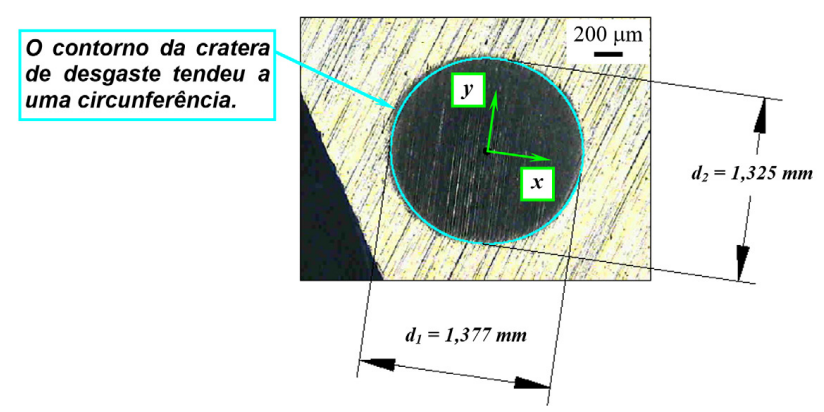

Figura 5. Ensaio realizado em um corpo-de-prova de aço-ferramenta M2. Contorno da cratera de desgaste tendendo a uma circunferência.

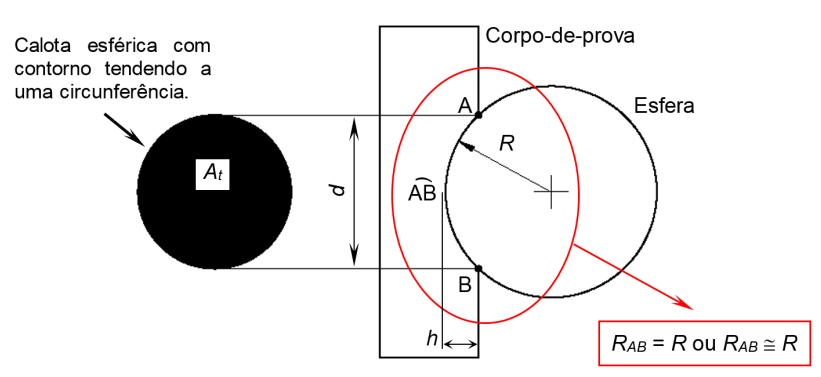

Figura 6. Quando $R_{A B}=R$ ou $R_{A B} \cong R$, o contorno da cratera de desgaste tenderá a uma circunferência.

\subsubsection{Contorno elíptico}

Em relação aos corpos-de-prova de WC-Co P20, no "3० Grupo de Ensaios", todas as crateras de desgaste apresentaram contornos com tendência a uma elipse, como exemplifica a Figura 7.

A partir de um determinado grau de desgaste, as crateras apresentaram contornos com tendência a uma elipse, influenciando nos cálculos das dimensões da cratera de desgaste. Uma precisa circularidade conduz a valores confiáveis de $h$ (profundidade), $V$ (volume) e $A_{t}$ (área projetada), caso essas grandezas sejam calculadas em função de $d$. Por outro lado, além da própria medição do diâmetro, a má formação da cratera de desgaste (caracterizada pelo contorno tendendo a uma elipse) acarreta cálculos errôneos de suas dimensões [15].

Conforme os ensaios vão sendo realizados, a esfera, inevitavelmente, vai sofrendo desgaste. Isso faz com que o 
arco $\overparen{\mathrm{AB}}$ não tenha mais o mesmo raio da esfera. $\bigcirc$ novo raio de $\overparen{\mathrm{AB}}$ será $R_{\mathrm{AB}}$ ', com $R_{\mathrm{AB}}{ }^{\prime}>R$, e o contorno da cratera de desgaste tenderá a uma elipse, como mostra a Figura 8.

Analisando-se a área elíptica de diagonais " $d$," e “ $\mathrm{d}_{2}$ " da Figura 8, observa-se que é possível obter diversos valores de $d$, com $d_{2} \leq d \leq d_{1}$. Como a área $A_{t}$ pode ser calculada em função de $d$, se $d=d_{1}$, será obtido um valor $\operatorname{de} A_{t}$ maior que o real e, se $d=d_{2}$, um valor menor. Como $h$ é determinado em função de $d$, o valor dessa dimensão também será impreciso. Além disso, pela Figura 8, nota-se ainda que $h_{\mathrm{el}}<h$, onde $h_{\mathrm{el}}$ é a altura da cratera de desgaste quando seu contorno tende a uma elipse e, $h$, quando tende a uma circunferência. Em relação a $V$, como essa dimensão pode ser calculada tanto em função de d (Equação I [ I6]), quanto em função $h$ (Equação 2 [16]), consequentemente será obtido um resultado inexato.

$$
\begin{aligned}
& V=\frac{\pi \cdot d^{4}}{64 R} \\
& h=\frac{d^{2}}{8 R}
\end{aligned}
$$

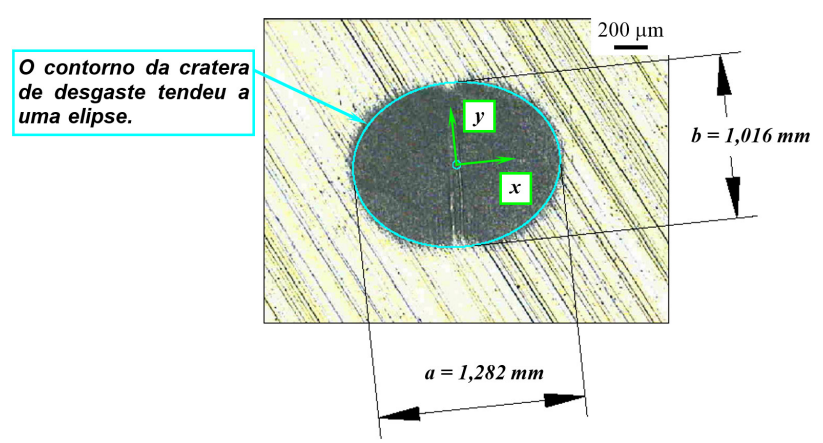

Figura 7. Ensaio realizado em um corpo-de-prova de WC-Co P2O. Contorno da cratera de desgaste tendendo a uma elipse.

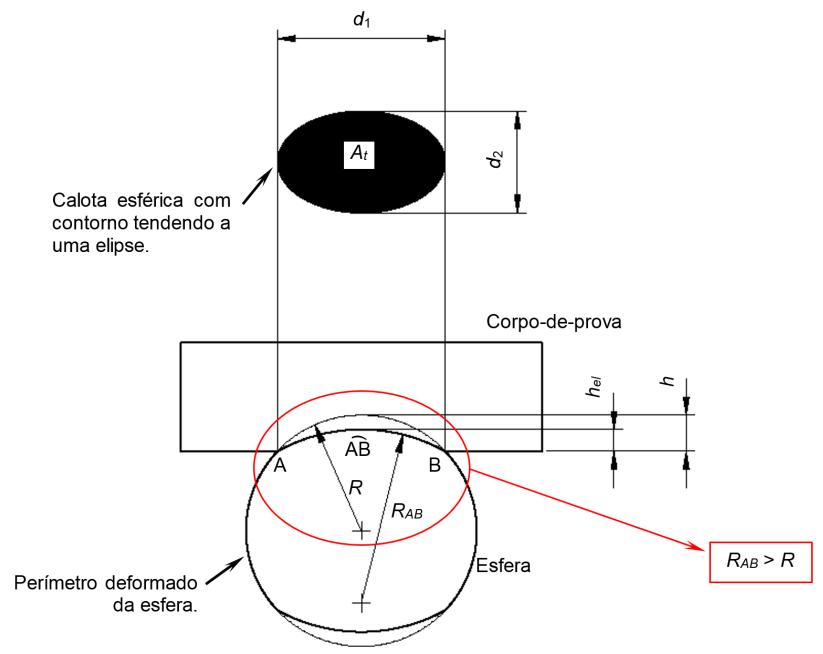

Figura 8. Quando $R_{A B}>R$, o contorno da cratera de desgaste tenderá a uma elipse.

\section{I.3 Contorno oblongo}

Os resultados das Figuras 5 e 7 mostraram exemplos em que $R_{\mathrm{AB}}=R$ (ou, $R_{\mathrm{AB}} \cong R$ ) e $R_{\mathrm{AB}}$ ' $>R$. Ainda no " $3^{\circ}$ Grupo de Ensaios" e, consequentemente, no " $4^{\circ}$ Grupo de Ensaios", foi reportado o contorno denominado de "oblongo", como exemplifica a Figura 9.

Essa situação ocorre quando $R_{A B}{ }^{\prime}>>>R$, como esquematizado na Figura 10. Nesse caso, a esfera de ensaio vai estar com um nível de desgaste mais elevado do que na situação em que $R_{A B}{ }^{\prime}>R$.

Nos ensaios realizados com os corpos-de-prova de aço-ferramenta $M 2$, as crateras de desgaste não apresentaram nenhum contorno semelhante ao "oblongo". Este resultado é justificável, pois, quando os ensaios foram conduzidos nos corpos-de-prova de HSS M2, a esfera não estava com o grau de desgaste necessário para gerar crateras com contornos semelhantes ao da Figura 9.

\subsubsection{Contorno " 8 "}

No "5" e último "Grupo de Ensaios", realizados nos corpos-de-prova de WC-Co P20, a esfera que já apresentava um grau de desgaste relativamente elevado, gerando crateras de desgaste com contorno denominado "8" ("formato oito"). A Figura I I exemplifica tal formato.

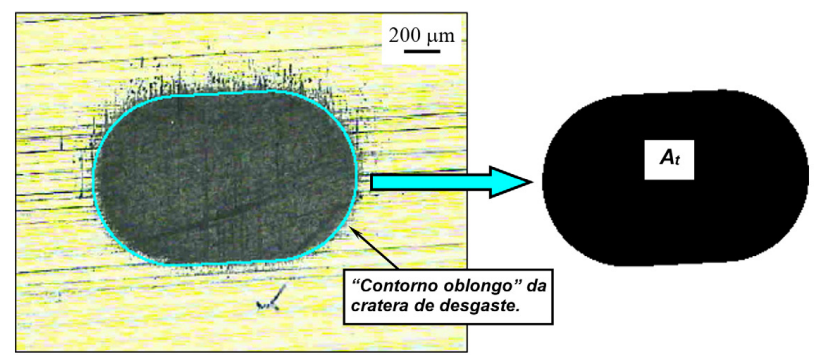

Figura 9. "Contorno oblongo" apresentado pela cratera de desgaste gerada em um corpo-de-prova de WC-Co P20.

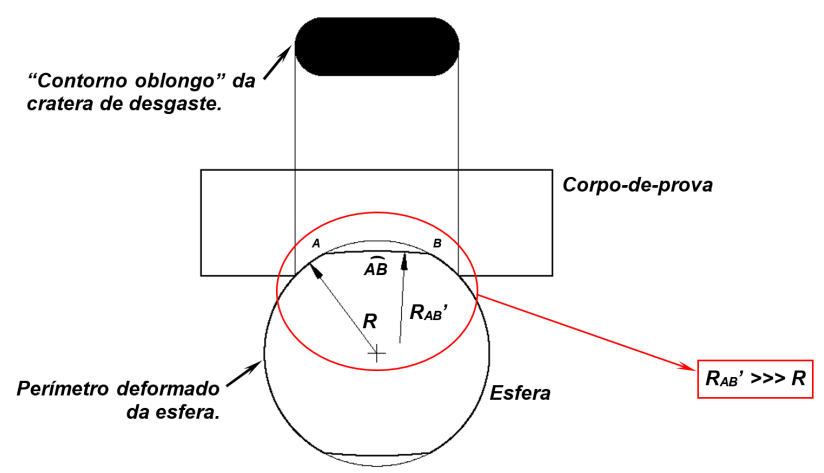

Figura 10: Caso em que $R_{A B}$ ' $>>R$. "Contorno oblongo" da cratera de desgaste. 


\subsection{Alterações dos Contornos das Crateras de Desgaste}

Durante os ensaios realizados com os corpos-de-prova de HSS M2 e WC-Co P20, foram observados diferentes contornos das crateras de desgaste, como mostra a Figura 12.

Conforme $\Xi$ foi aumentando, os contornos das crateras de desgaste foram se alterando. Quando $\Xi$ estava

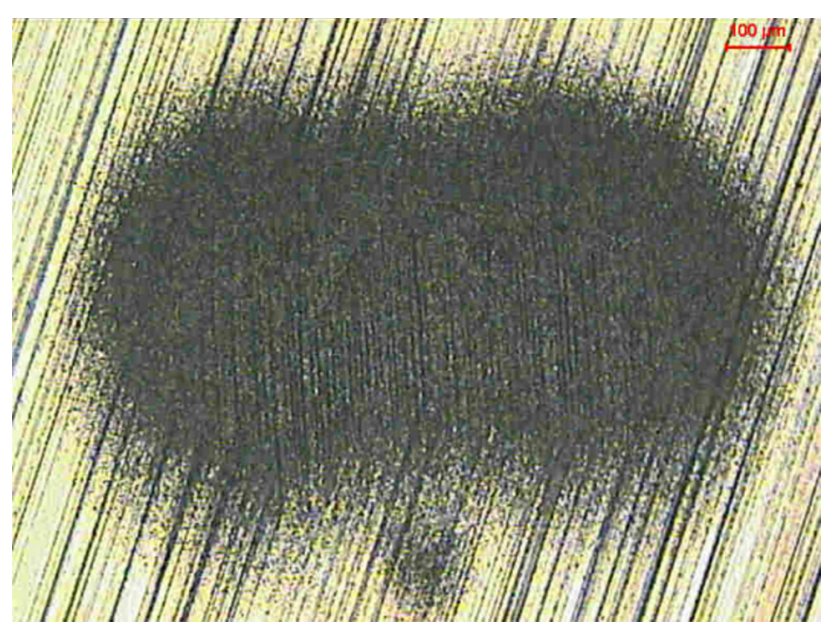

Figura I I. "Contorno 8" apresentado pela cratera de desgaste gerada em um corpo-de-prova de WC-Co P20. relativamente baixo, os contornos tenderam a uma circunferência ("contorno circunferencial"). Com o aumento do nível de desgaste, os contornos começaram a tender para uma elipse ("contorno elíptico"). A partir de um determinado nível de desgaste da esfera, as crateras apresentaram contornos aqui denominados de "contorno oblongo". No nível mais elevado de desgaste atingido, a esfera gerou crateras com contornos semelhantes ao número 8 (oito). Esse contorno foi designado de "contorno 8".

Com base nos quatro contornos relatados, o desgaste da esfera foi classificado em quatro níveis $\Xi$ distintos, conforme a Figura 13. Embora tenham sido atribuídos valores numéricos a $\Xi$, o mesmo continua sendo apenas um parâmetro qualitativo, não possuindo qualquer unidade.

\subsection{Relação Entre o Desgaste da Esfera e o Desvio-Padrão de $d_{\text {médio }}$}

Inicialmente, serão discutidos os resultados obtidos com o HSS M2 e, em seguida, os resultados referentes aos corpos-de-prova de WC-Co P20.

Neste item, $\sigma_{P}$ refere-se ao desvio-padrão de $d_{\text {médio }}$ (média aritmética das quatro medidas de $d$, para cada distância de deslizamento). No caso de crateras com contornos elíptico ou oblongo, a medida $d$ corresponde ao maior diâmetro. Em relação ao "Formato 8", não foram calculadas quaisquer grandezas relacionadas $\left(d, V\right.$ ou $\left.A_{t}\right)$,

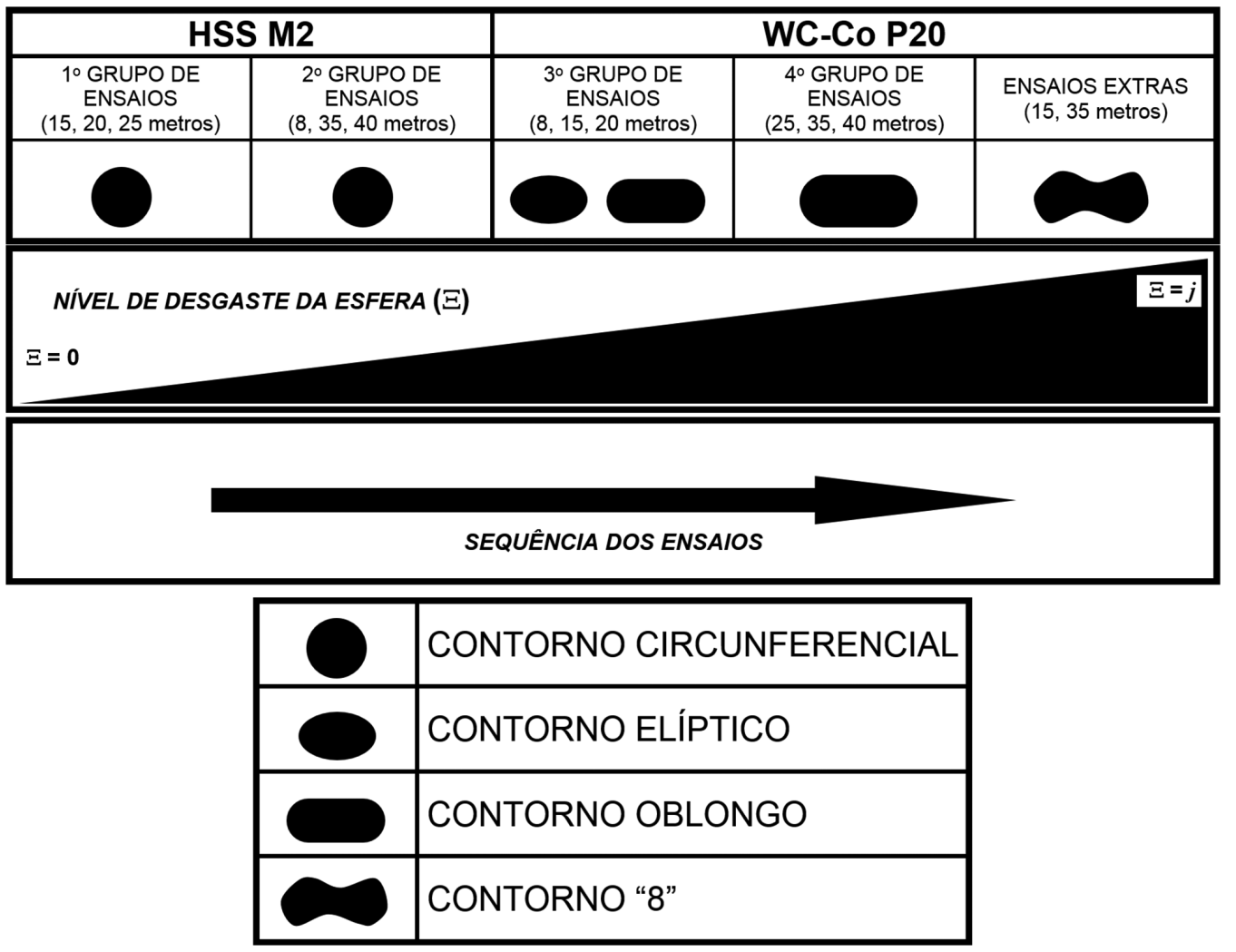

Figura 12. Ordem de alterações dos contornos das crateras de desgaste. 
devido aos erros relativamente elevados inseridos nos cálculos. Na Tabela 3 estão os valores de $d_{\text {médio }}$ e $\sigma_{p}$, obtidos com o aço ferramenta $\mathrm{M} 2$ e com o WC-Co P20.

A Figura 14 mostra a relação de $\sigma_{P} \operatorname{com} \Xi$, para os ensaios realizados com o HSS M2 e com o WC-Co P20. Para melhorar a escrita do texto, o termo "nível de desgaste da esfera”, algumas vezes será denotado pelo símbolo grego “ $\Xi$ ”.

Neste trabalho, convencionou-se que, no início dos ensaios com o HSS M2, $\Xi=0$ e, no final, $\Xi=i$. Logo, é correto afirmar que, com o WC-Co P20, a esfera iniciou

Tabela 3. Corpos-de-prova de HSS M2 e WC-Co P20. Valores de $d_{\text {médio }}$ e $\sigma_{p}$

\begin{tabular}{|c|c|c|c|c|c|c|}
\hline$S[\mathrm{~m}]$ & & $d_{\text {médio }}[\mathrm{mm}]$ & $\sigma_{p}[\mathrm{~mm}]$ & & $d_{\text {médio }}[\mathrm{mm}]$ & $\sigma_{p}[\mathrm{~mm}]$ \\
\hline 8 & & 1,068 & 0,037 & & $\mathrm{I}, 367$ & 0,110 \\
\hline 15 & & 1,360 & 0,016 & 0 & $\mathrm{I}, 443$ & 0,128 \\
\hline 20 & $\mathfrak{s}$ & $|, 4| 8$ & 0,018 & $\tilde{n}$ & 1,439 & 0,131 \\
\hline 25 & 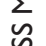 & 1,469 & 0,015 & ن & 1,918 & 0,062 \\
\hline 35 & 吕 & $\mathrm{I}, 444$ & 0,049 & $u_{3}^{\prime}$ & 1,909 & 0,036 \\
\hline 40 & & 1,963 & 0,039 & & I,867 & 0,059 \\
\hline
\end{tabular}

\begin{tabular}{|c|c|c|c|}
\hline $\begin{array}{c}\text { FORMATO } \\
\text { CIRCUNFERENCIAL }\end{array}$ & FORMATO ELÍPTICO & FORMATO OBLONGO & FORMATO "8" \\
\hline $\boldsymbol{\Xi}=1$ & $\boldsymbol{E}=\mathbf{2}$ & $\boldsymbol{\Xi}=\mathbf{3}$ & $\boldsymbol{\Xi}=\mathbf{4}$ \\
\hline
\end{tabular}

NÍVEL DE DESGASTE DA ESFERA ( $\Xi)$

Figura 13. Classificação dos níveis de desgaste $\Xi$ da esfera, em função do contorno da cratera de desgaste.

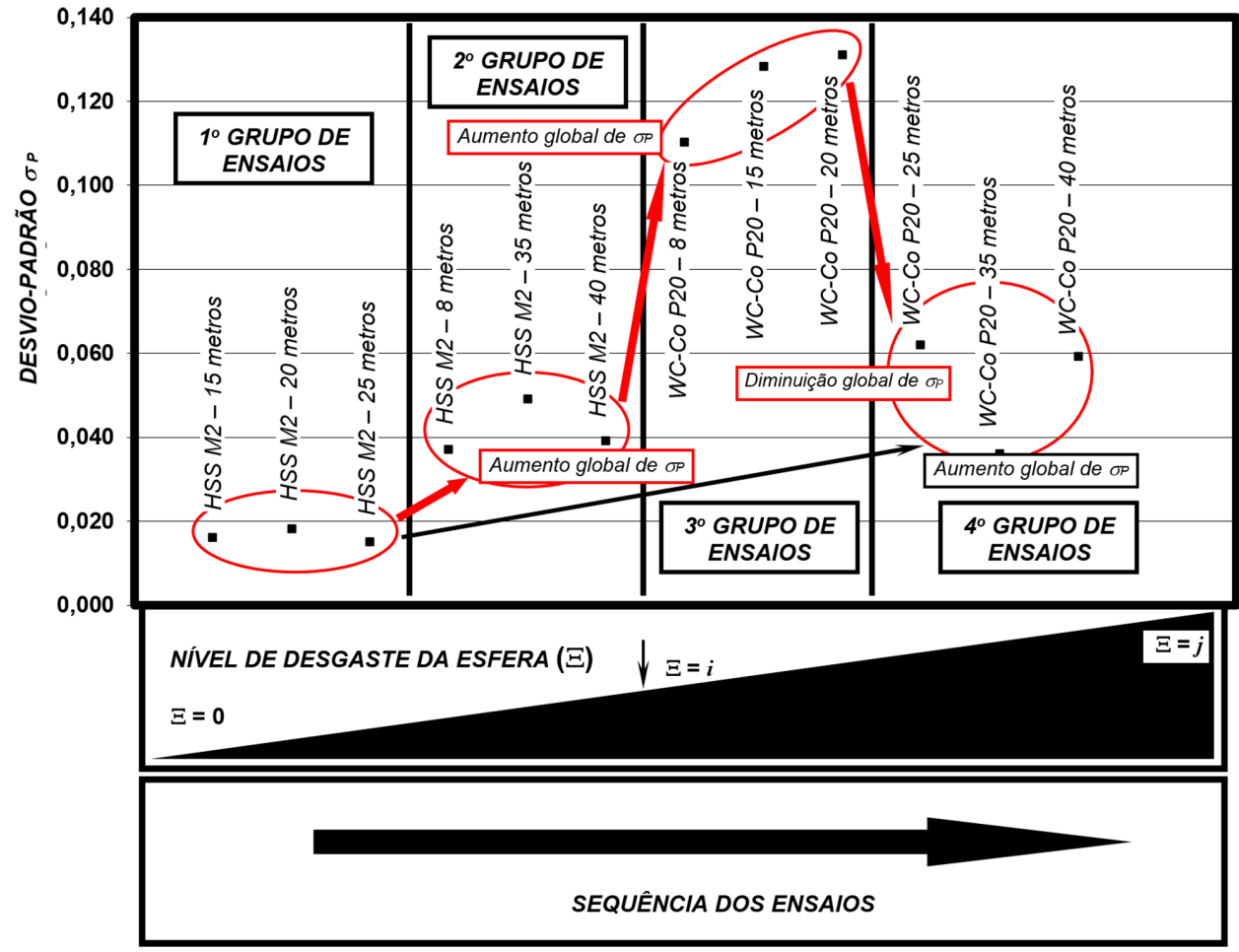

Figura 14. Corpos-de-prova de HSS M2 e WC-Co P20. Gráfico de $\sigma_{p}$ em função de $\Xi$. 
os ensaios com $\Xi=i$, e terminou com um nível maior de desgaste, $\Xi=j$. “i” e "j" são referências qualitativas, adotadas para indicar que, "no final dos ensaios, a esfera está com um nível de desgaste maior que no início". Matematicamente, é correto escrever que $\boldsymbol{j}>\boldsymbol{i}$.

Analisando-se os dois primeiros grupos de ensaio (HSS M2), nota-se que, todos os valores de $\sigma_{p}$ do $l^{\circ}$ grupo, foram menores que os do $2^{\circ}$. Com os corpos-de-prova de WC-Co P20 ( $3^{\circ}$ e $4^{\circ}$ grupos), foram obtidos desvios-padrão relativamente elevados, quando comparados aos valores relatados para o HSS $\mathrm{M} 2$.

Globalmente, para os $1^{\circ}, 2^{\circ}$ e $3^{\circ}$ grupos, conforme os ensaios foram sendo realizados e, fatalmente o desgaste da esfera foi aumentando, os valores de $\sigma_{P}$ aumentaram. Somente $\circ 4^{\circ}$ grupo não acompanhou essa tendência.

Pelos resultados obtidos, observa-se que $\Xi$ exerce influência sobre $\sigma_{p}$, e que $\sigma_{p}$ tende a aumentar com o aumento de $\Xi$.

\section{CONCLUSÕES}

Em função dos resultados obtidos, pode-se concluir que:

- Em ensaios de desgaste micro-abrasivo por esfera rotativa, deve-se produzir crateras com contornos circunferenciais, a fim de se obter, em termos de geometria da calota, resultados precisos de $d, h$ e $V$;

- Entretanto, em função do nível de desgaste da esfera de ensaio, poderão ser formadas crateras de desgaste com contornos que divergem do formato circunferencial, que neste trabalho foram classificados como "contorno elíptico", "contorno oblongo" e “contorno 8";

- Com o aumento do grau de desgaste da esfera de ensaio, o desvio-padrão dos valores dos diâmetros das crateras poderão aumentar.

\section{REFERÊNCIAS}

I Cozza RC, Mello JDB, Tanaka DK, Souza RM. Relationship between test severity and wear mode transition in micro-abrasive wear tests. Wear. 2007;263(I-6): I I I- I I6. http://dx.doi.org/ I0. I0 I6/j.wear.2007.0 I.099.

2 Cozza RC, Tanaka DK, Souza RM. Friction coefficient and abrasive wear modes in ball-cratering tests conducted at constant normal force and constant pressure - Preliminary results. Wear. 2009;267( I-4):6 I-70. http://dx.doi. org/10.1016/j.wear.2009.01.055.

3 Trezona RI, Allsopp DN, Hutchings IM. Transitions between two-body and three-body abrasive wear: influence of test conditions in the microscale abrasive wear test. Wear. 1999;225-229:205-2 I4. http://dx.doi.org/I0.1016/S00431648(98)00358-5.

4 Batista JCA, Matthews A, Godoy C. Micro-abrasive wear of PVD duplex and single-layered coatings. Surface and Coatings Technology. 200 I; |42-I44: I I37-I |43. http://dx.doi.org/ I0.10 I6/S0257-8972(0 I)0 I I89-6.

5 Batista JCA, Godoy C, Matthews A. Micro-scale abrasive wear testing of duplex and non-duplex (single-layered) PVD (Ti,Al)N, TiN and Cr-N coatings. Tribology International. 2002;35(6):363-372. http://dx.doi.org/l0.1016/ S030I-679X(02)000 I 7-8.

6 Cozza RC. A study on friction coefficient and wear coefficient of coated systems submitted to micro-scale abrasion tests. Surface and Coatings Technology. 201 3;2 I 5:224-233. http://dx.doi.org/l0.10 I6/j.surfcoat.2012.06.088.

7 Cozza RC. Effect of pressure on abrasive wear mode transitions in micro-abrasive wear tests of WC-Co P20. Tribology International. 2013;57:266-27I. http://dx.doi.org/10.10I6/j.triboint.2012.06.028.

8 Adachi K, Hutchings IM. Wear-mode mapping for the micro-scale abrasion test. Wear. 2003;255(I-6):23-29. http:// dx.doi.org/10.1016/S0043-1648(03)00073-5.

9 Adachi K, Hutchings IM. Sensitivity of wear rates in the micro-scale abrasion test to test conditions and material hardness. Wear. 2005;258(I-4):3 I8-32I. http://dx.doi.org/10.1016/j.wear.2004.02.016.

10 Cozza RC. Estudo do comportamento do coeficiente de desgaste e dos modos de desgaste abrasivo em ensaios de desgaste micro-abrasivo [dissertação de mestrado]. São Paulo: Universidade de São Paulo; 2006 [acesso em 22 jul. 20l4]. Disponível em: http://www.teses.usp.br/.

I I Ramalho A. Micro-scale abrasive wear of coated surfaces-prediction models. Surface and Coatings Technology. 2005; 197(2-3):358-366. http://dx.doi.org/10.10 I6/j.surfcoat.2004.12.024. 
12 Cozza RC. Estudo do desgaste e atrito em ensaios microabrasivos por esfera rotativa fixa em condições de força normal constante e pressão constante [tese de doutorado]. São Paulo: Universidade de São Paulo; 201 I [acesso em 22 jul. 20l4]. Disponível em: http://www.teses.usp.br/.

13 Gee MG, Gant AJ, Hutchings IM, Kusano Y, Schiffman K, Van Acker K, et al. Results from an interlaboratory exercise to validate the micro-scale abrasion test. Wear. 2005;259(I-6):27-35. http://dx.doi.org/10.1016/j.wear.2005.02.092.

14 Izhevskyi VA, Genova LA, Bressiani JC, Bressiani AHA. Liquid phase sintered SiC ceramics from starting materials of different grade. Cerâmica. 2004;50(3I5):26I-267. http://dx.doi.org/I0.1590/S0366-69I320040003000I4.

15 Cozza RC, Souza RM, Tanaka DK. Influência do desgaste da esfera na formação das calotas em ensaios de desgaste micro-abrasivo por esfera rotativa fixa. In: Anais do IV Congresso Nacional de Engenharia Mecânica - CONEM 2006. Recife; 2006.

16 Rutherford KL, Hutchings IM. Theory and application of a micro-scale abrasive wear test. Journal of Testing and Evaluation. 1997;25(2):250-260. http://dx.doi.org/I0. I520/JTE I |487J.

Recebido em: 5 Out. 2014

Aceito em: 27 Mai. 2015 